\title{
Ovine fetuses from slaughterhouses: A useful source for neural cell primary cultures
}

\author{
Vittorio Farina, Sergio Domenico Gadau, Gianluca Lepore, Marcella Carcupino, Marco Zedda \\ Department of Veterinary Medicine, University of Sassari, Sassari, Italy
}

\begin{abstract}
A lot of evidence demonstrates that sheep could represent an experimental model to set up medical procedures in view of their application on humans. Sheep are chosen as models for human biomechanical studies because their skeleton has some similarities to humans. The aim of this work was to set up sheep primary cultures from ovine fetuses at different ages, from pregnant uteri retrieved at local abattoirs. Cell characterization showed that one cell population was immunopositive to GFAP and identifiable as astrocytes, whereas a second cell type was III $\beta$-tubulin-positive, and hence classified as neurons. At 60-days old fetus is suitable to obtain neurons, whereas in a 90-days old fetus the cell culture is
\end{abstract}

Correspondence: Marco Zedda, Department of Veterinary Medicine, University of Sassari, Via Vienna 2, 07100 Sassari, Italy.

E-mail: mzedda@uniss.it

Key words: Sheep fetus; primary glial and cell cultures; confocal microscopy; 3Rs statement.

Acknowledgements: The authors wish to thank Dr. G. Finlayson and Dr. N. Giordano for their review of the English manuscript.

Contributions: VF and MZ designed the project; SDG and GL designed the project and performed the experimental procedures; MZ, GL and SDG performed the statistical analyses and wrote the paper; MC prepared the table and figures.

Conflict of interest: The authors declare no conflict of interest.

Ethics statement: We declare that the animals used in this work are not live animals but taken at Public Slaughterhouse and therefore our procedures have not passed through the examination of the Department Ethics Committee. Indeed, fetuses were obtained at a maximum 90 day of gestation, a period considered still very early to take into account from the Department Ethics Committee.

Received for publication: 10 September 2020.

Accepted for publication: 7 Aprile 2021.

${ }^{\circ}$ Copyright: the Author(s), 2021

Licensee PAGEPress, Italy

Journal of Biological Research 2021; $94: 9344$

doi:10.4081/jbr.2021.9344

This article is distributed under the terms of the Creative Commons Attribution Noncommercial License (by-nc 4.0) which permits any noncommercial use, distribution, and reproduction in any medium, provided the original author(s) and source are credited. predominantly characterized by glial cells. The procedure here proposed is inexpensive, in fact, collecting fetuses during sheep slaughtering is a cost-saving option, unlike common experimental animals such as mice, rats, rabbits, that require very high economical efforts. Finally, our protocol fully eliminates the need of animal killing, being living animals replaced by a validated in vitro model in agreement with the 3 Rs statement.

\section{Introduction}

In experimental biology, the need to turn to an experimental model that might be closer to humans is increasingly pressing. Indeed, although rodents remain the most widely used species for experimental research, other mammals may be useful because of their complex anatomy and physiology in comparison to rodents. Therefore, several papers have been published in order to underline the validity of other animal models such as dogs and cattle. ${ }^{1-3}$ Recently, sheep tissues and organs have been used to perform experimental procedures in human pathological conditions studies. Scientific works carried out on sheep are very numerous and with different aims due to the fact that these animal species show similarities to humans in lots of anatomical, physiological and pathological features. By browsing the concerning literature, it can be noted that almost all anatomical systems of sheep have been considered as useful experimental models to set up medical procedures in a view of their application on humans. Sheep are chosen as a model for human biomechanical studies because their skeleton has similarities with humans, for example in mineral metabolism, bone density and bone healing. ${ }^{4}$ Sheep are also frequently used for preclinical analyses as they represent a very useful model to assess safety and pharmacokinetics of microbicide drugs. ${ }^{5}$

In accordance with the literature there are many other advantages of using sheep as an experimental model. In neuroscience studies, sheep have acquired an increasing interest for many aspects. It is worth to note that this species is a spontaneous model for the formation of neurofibrillary tangles in the cerebral cortex, which are typical of neurological disorders, such as Alzheimer's disease. ${ }^{6,7}$ There are many advantages in studying the cerebral cortex of sheep as it has cerebral gyri similar to those in the human neocortex. The presence of gyri and sulci in sheep brain provides valuable signposts to identify specific cortical regions, unlike rats or mice that are lissencephalic animals. ${ }^{8}$ Moreover, the size of the sheep brain may facilitate physiological and neuropathological comparisons with human brain. Sections of sheep hypothalamus have been successfully studied as a model of brain sex differentiation. In particular, the morphology of the ovine sexually dimorphic nucleus, located in the central region of the preoptic area of the hypothalamus, has been examined by several authors which 
found interesting results applicable to many mammalian species including humans. ${ }^{9,10}$ Cell cultures carried out from sheep brain represent another important tool for studying neural cell biology instead of the usual cell cultures obtained from rats. ${ }^{11-15}$ Actually, in addition to ovine cultured cells, human-derived cell models exist, including stem cell-derived primary neuronal and glial cultures, as well as sophisticated 3D organoids. ${ }^{16-18}$ However, such models may be expensive and/or need a high degree of manipulation in order to get differentiated neural cells.

The aim of this work is to evaluate different options in setting up sheep neuronal or glial primary cultures from fetuses collected in slaughterhouses otherwise intended for destruction, with no animal suffering and without using laboratory animals.

\section{Materials and Methods}

\section{Sampling}

Fetuses at different ages were collected in local slaughterhouses (Figure 1). Sheep were healthy and regularly slaughtered since pregnancy was not known to the farmers. As a matter of fact, during spring-summer period there are good chances to find accidentally pregnant sheep in Sardinian public slaughterhouses, as extensive farming is conducted in Sardinia and therefore sheep are in continuous presence of the ram. In that period, about $10 \%$ of sheep may be found casually pregnant. As far as the present study is concerned, the sampling took place on pregnant dead sheep whose fetuses were destined for disposal, so that neither anaesthesia nor euthanasia were performed.

Within half an hour after collection the fetuses were transported to our laboratory at $39^{\circ} \mathrm{C}$, which is the basal temperature of this species. ${ }^{19}$ Once at the laboratory, the uteri were opened under sterile conditions, and fetuses were removed through fetal adnexa dissection. First of all, the period of gestation was estimated on the base of the fetal crown-rump length following the tables reported in the literature. ${ }^{20}$ Based on these measurements, fetuses at three different gestation ages (40, 60, 90 days) were collected. By using a stereo microscope each head was dissected in order to remove brain tissue eliminating the meninges as much as possible.

Five brain tissues for each gestational period were collected, for a total of fifteen brains. Homogenates were mechanically

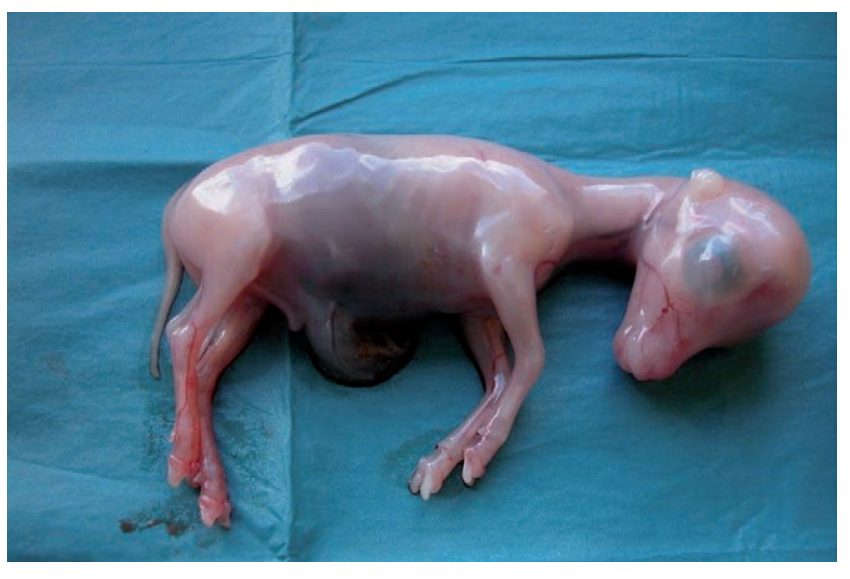

Figure 1. Ovine embryo at $\mathbf{4 0}$ gestation days. The period of gestation was estimated on the base of the fetal crown-rump length. obtained (Turrax, IKA, Staufen, Germany) from each brain tissues and then suspended in an ice-cold freezing medium containing Dulbecco's Modified Eagle's Medium (DMEM), 1\% HEPES (pH 7.4), $1 \%$ fetal calf serum and 10\% dimethylsulfoxide (DMSO). Tissues were then stored in liquid nitrogen until use.

\section{Cell culture procedures}

Frozen tissues were rapidly thawed in a water bath at $39^{\circ} \mathrm{C}$ and minced in small fragments under sterile conditions into a laminar flow hood. A papain dissociation kit (Worthington Biochemical Corporation, Lakewood, NJ, USA) was used to dissociate cells following the company guidelines. Cells were then suspended in a basal medium consisting of 1:1 DMEM and Ham's F12, supplemented with penicillin $(30 \mathrm{mg} / \mathrm{L})$, streptomycin $(50 \mathrm{mg} / \mathrm{L})$, sodium bicarbonate $(2.4 \mathrm{~g} / \mathrm{L})$, insulin $(10 \mu \mathrm{g} / \mathrm{mL})$, transferrin $(10 \mu \mathrm{g} / \mathrm{mL})$, sodium selenite $\left(10^{-8} \mathrm{M}\right)$ and $10 \%$ fetal calf serum, at $39^{\circ} \mathrm{C}$ in a $5 \%$ $\mathrm{CO}_{2}$ humidified atmosphere. Cells were plated at the rate of $5 \times 10^{5}$ on glass coverslips, previously coated with poly-L-lysine. All reagents, when not specified, were from Merck, (Darmstadt, Germany).

\section{Immunofluorescence staining}

In order to distinguish neurons and astrocytes, a double immunofluorescence was performed following the methodology previously illustrated. ${ }^{3,21}$ Briefly, at day 10 of culture, cells were fixed with $4 \%$ paraformaldehyde at room temperature for $30 \mathrm{~min}$, washed with PBS, and then permeabilized with $0.1 \%$ Triton X-100 for $15 \mathrm{~min}$ at room temperature. Subsequently they were incubated overnight with anti-class III $\beta$-tubulin antibody (monoclonal, clone DM1A), a widely-recognized marker of neuronal cells (Dráberová et al. 1998) $)^{22}$ diluted $1: 200$, and then for $1 \mathrm{~h}$ at $37^{\circ} \mathrm{C}$ with anti-mouse FITC-conjugated antibody diluted 1:100 (AlexaFluor 488, Termo Fisher, Waltham, MA, USA). Cells were then incubated for $1 \mathrm{~h}$ with polyclonal anti-GFAP antibody, diluted 1:200, specific for glial fibrillary acidic protein present in astrocytes. Finally, an incubation of $1 \mathrm{~h}$ with a secondary anti-rabbit TRITC-conjugated antibody, diluted 1:100 (AlexaFluor 594) was carried out. In addition, nuclei were counterstained with Hoechst \#33258 fluorescent dye. Additional monolayers were used as negative control by omitting the primary antibody or employing non-immune mouse serum. Cells were finally observed with a Leica TCS SP5 DMI 6000CS confocal microscope (Leica Microsystems GmbH, Wetzlar, Germany). FITC was excited at $488 \mathrm{~nm}$ and emission was detected between 510 and $550 \mathrm{~nm}$ whereas TRITC was excited at $568 \mathrm{~nm}$ and emission was detected between 585 and $640 \mathrm{~nm}$. Microscopic pictures of monolayers were taken, each including more than 50 cells. In order to evaluate the number of neurons and astrocytes, we proceeded as follows: five high-magnification photos acquired by confocal microscope were taken for each monolayer (15 in total). A grid composed of twelve squares $\left(387 \mu \mathrm{m}^{2}\right)$ was placed on each picture using ImageJ freeware analysis software. Through the Analyze/Counter plugin, we proceeded to count the cells which were positive to anti- $\beta$ III tubulin antibody (neurons) and then those positive to anti-GFAP (astrocytes). Cell number was expressed in percentage. The counting was repeated by three different researchers to evaluate possible interobserver differences, which were lower than $2 \%$.

\section{Statistics}

All data was expressed as means $\pm \mathrm{SD}$ and tested by One-Way ANOVA. Statistical analyses were performed by using Statigraphics software (Statigraphics Technologies, Inc., The Plains, VA, USA) and a $\mathrm{p}<0.05$ was considered significant. 


\section{Results}

Technical procedures led to setting up neural primary cultures starting from all the gestation periods taken into consideration $\left(40^{\text {th }}, 60^{\text {th }}\right.$ and $90^{\text {th }}$ day), obtaining several proliferating cells. At gestational days 60 and 90 cells were $40 \%$ and $110 \%$, respectively, more numerous than at gestational day 40 (Figure 2). Cell plates from the five brain fragments belonging to the same gestational period were homogenous as to morphology and growth rate after 10 days of culture.

Two cell types were mostly observed: i) stellate cells showing a pleomorphic shape, large flat cell bodies and cytoplasmic processes, ii) cells with spindle-like or triangular-shaped cell bodies, with long processes that tended to have contacts with those of contiguous cells. The immunofluorescence characterization of these two cell populations testified that the first cell-type was immunopositive to GFAP and thus identifiable as astrocytes, whereas the second type was formed by neurons as they were III $\beta$-tubulin-positive (Figure 3). Although both cell types were present at all three scheduled times, the two populations showed different percentages as reported in Figure 3. Specifically, at day 40 of pregnancy cultured cells were prevalently neurons $(60 \%)$ and at the day 60 , neurons were even more numerous $(90 \%, \mathrm{p}<0.0001)$. On the other hand, at the day 90 the most numerous cells were astrocytes $(80 \%, \mathrm{p}<0.001)$. Differences in astrocyte number at 40 and 60 days and the number of neurons at 90 days were not statistically significant.

\section{Discussion}

Primary neural cell cultures represent a useful protocol to study cell morphology, cell physiology and toxicology. ${ }^{23}$ Recently, the research of experimental models suitable to humans has increased. Indeed, the favourite animal models are those showing brain size, brain complexity, life span expectancy and diseases similar to those affecting humans. ${ }^{24,25}$ Human-derived cell cultures exist, including stem cell-derived primary neuronal and glial cultures, as well as sophisticated 3D. ${ }^{16-18}$ However, cultures from different animal species are also exploited as validated models.

In this work, ovine fetuses at different developmental stages have been employed in order to obtain neural primary cell cultures with the aim to compare cultures set up from traditional laboratory animals. Our results show that sheep primary neural cell cultures are very useful as they allow to obtain significant amounts of viable neuronal and glial cells without difficulty. Furthermore, our work confirms the usefulness of the dissociation method with papain. Such enzyme allows a mild dissociation, giving the possi-
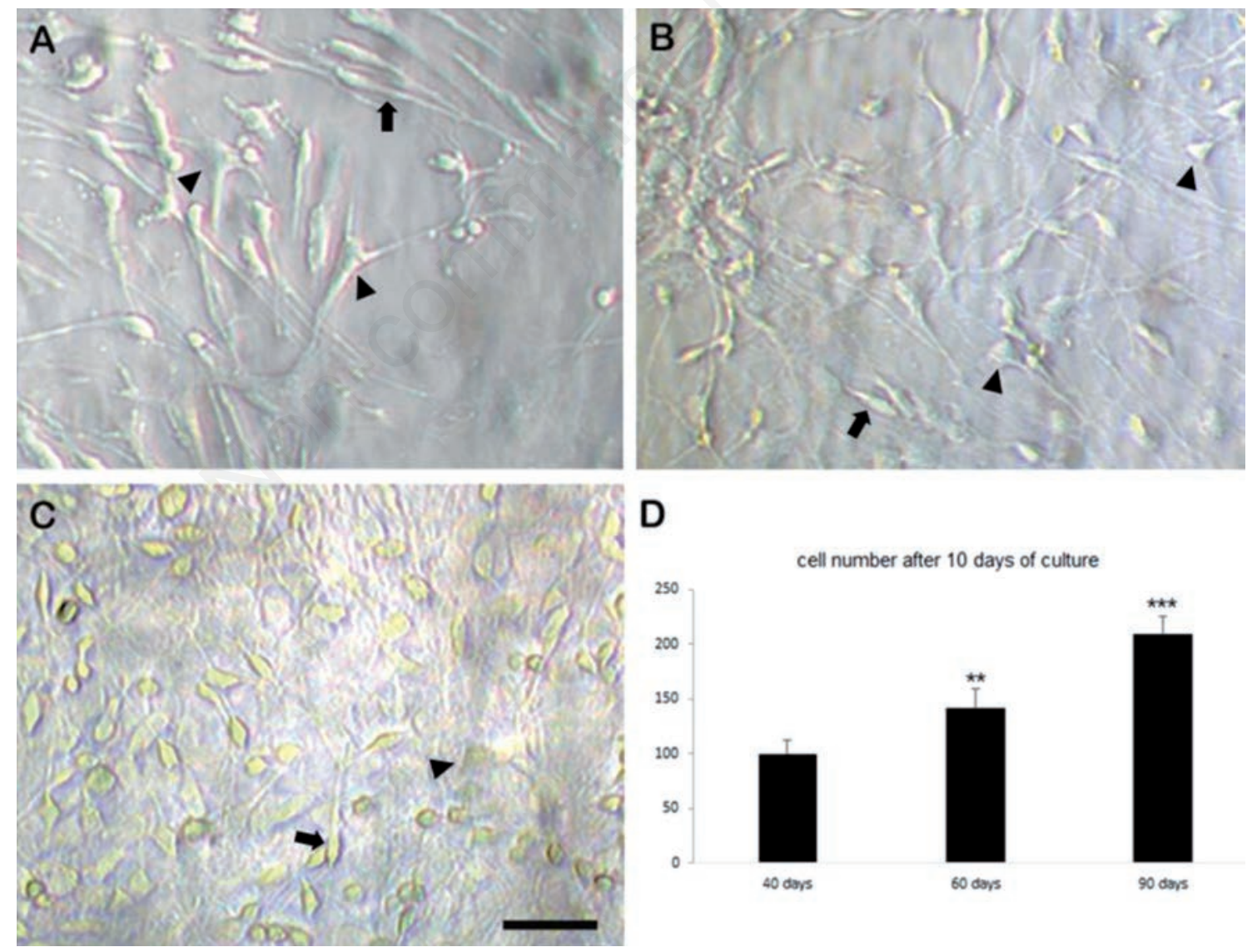

D

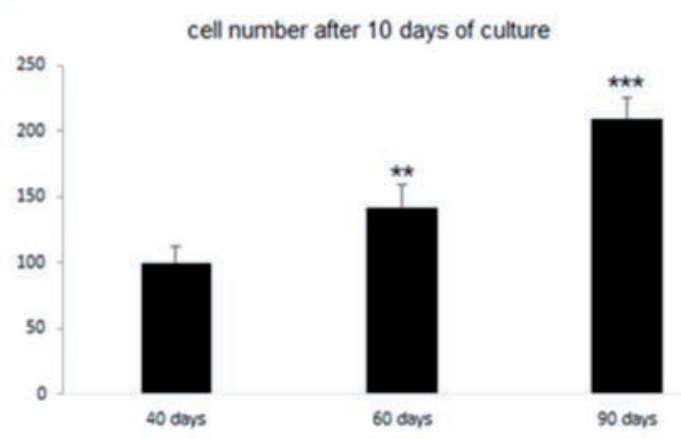

Figure 2. Phase contrast optics. Evaluations of primary cell cultures from fetal sheep brain at different development stages: 40 (A), 60 (B) and 90 (C) gestation days. Arrows indicate spindle shaped cells; arrowheads indicate stellate cells. Bar=60 $\mu \mathrm{m}$. The diagram (D) shows the different amount of cells after 10 days of culture. Data are expressed in percentage in comparison to the value of cell numbers from 40 days old fetuses. ${ }^{* *} \mathrm{p}<0.01,{ }^{* * *} \mathrm{p}<0.001$. 
bility to obtain a high number of viable neural cells. ${ }^{26}$ Moreover, as already highlighted, our experiment confirms the usefulness of the cryopreservation of explants, as it allows to obtain a proliferating culture even years after collection. ${ }^{1}$ Concerning the different gestational periods of fetuses from which the samples were taken, the data obtained demonstrates to be original. It is known that in rodents fetal age may affect the amount of neural cells obtainable while in addition our data provides information on ovine species. ${ }^{27}$ From data obtained in this work, it can be asserted that cell collect from fetal brains at day 60 of pregnancy is optimal if researchers wish to set up a primary culture where neurons are prevalent, whereas cell collect from fetal brains on the day 90 of pregnancy is suitable if a culture of astrocytes is required. Sometimes a coculture of neurons and astrocytes is needed, for example when the research is focused on the relationships between the two neural cell types. In this case, sheep fetuses represent a useful source of brain tissue, since a co-culture of neurons and astrocytes can be easily obtained from brains of fetuses at the day 40 of pregnancy. With no specific growth factors for glial or neuronal cells, but simply using fetuses at different gestation periods, different types of neural cells could be obtained.

Unlike other traditional laboratory animals, sheep fetuses in our protocol came from ewes intended to be slaughtered and hence no scheduled sacrifices involving living animals took place.

This protocol allows exploitation of biological material otherwise destined for destruction avoiding the use of experimental animals and their euthanasia, in accordance to the guidelines of the 3Rs Program. In the last decades, alternatives to animal testing have been proposed in order to enforce the strategy of 3Rs principles. ${ }^{28,29}$ Despite their large use, the experimental models based on
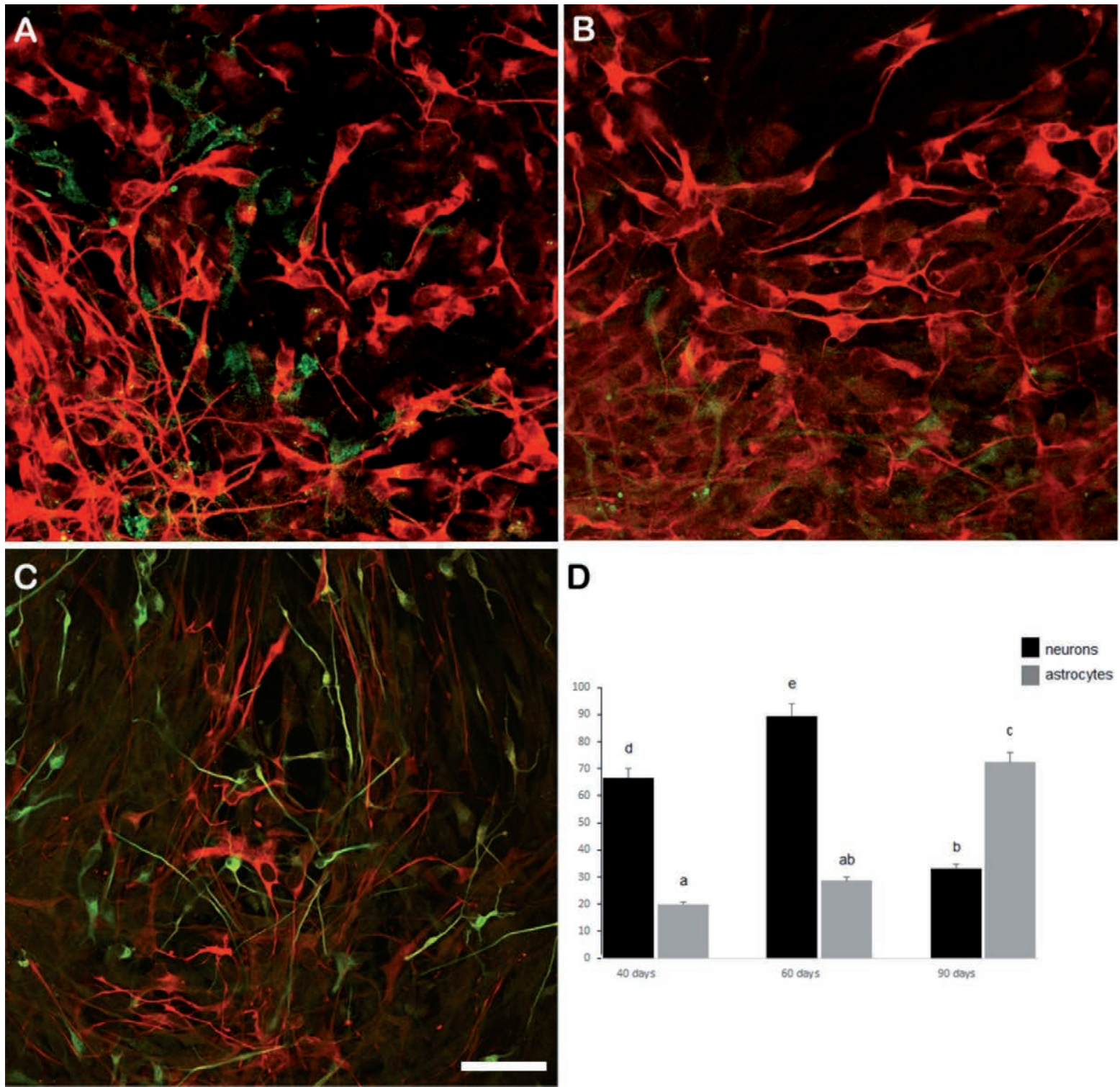

Figure 3. Double immunofluorescence staining. This method shows the two different cell populations: immunopositive neurons to anti$\beta$ III tubulin (red), and immunopositive astrocytes to anti-GFAP (green) on cultures belonging to fetal ovine brain explants of 40 (A), $60(B)$ and $90(C)$ days of gestation. Bar $=25 \mathrm{\mu m}$. The diagram (D) highlights the percentage of neurons and astrocytes at different stages of fetal development. ANOVA One Way. Lower case letters indicate statistical differences (c, d, e, p<0.0001). 
mice and rats show some limitations when compared to the investigations on large mammals, ${ }^{30}$ such as the inbred nature of certain rodent strains and a short life span. Therefore, various Authors have proposed several experimental models involving animal species closer to humans than mice and rats, such as dogs, cows and sheep. ${ }^{2}$ Currently the general trend towards saving animal lives in experimental studies is increasing, and animal welfare is becoming of primary importance at all experimental stages. The replacement of animals with a validated alternative method, such as in vitro culture, is suggested. The present protocol is consistent to the principle that the experimental model must be carefully chosen to ensure the analogy to humans, to avoid excessive use of animals and to prevent waste of time, effort and high costs. ${ }^{4}$

\section{Conclusions}

Our work highlights how the collection of non-marketable uteri of one domestic species can allow the preparation of a rigorous and effective protocol for the preparation of primary neural cultures, in full compliance with the principles of the 3Rs. Moreover, the in vitro procedure here described is inexpensive. On the other hand, the use of experimental animals such as mice, rats, etc., requires very high economical efforts, since they must be housed in suitable enclosures according to the law. The collection at the slaughterhouse followed by an adequate cryopreservation in liquid nitrogen of samples otherwise destined for destruction allows the availability of samples for the preparation of long-lasting cell cultures. Finally, our protocol fully eliminates the need of housing experimental animals, the latter being replaced by a validated in vitro model.

\section{References}

1. Hashimoto A, Onodera T, Ikeda H, Kitani H. Isolation and characterization of fetal bovine brain cells in primary culture. Res Vet Sci 2000;69:39-46.

2. Peruffo A, Cozzi B. Bovine brain: an in vitro translational model in developmental neuroscience and neurodegenerative research. Front in Pediatr 2014;2:74.

3. Gadau SD. Tubulin post-translational modifications in developing dog primary neurons obtained with methods according to the 3Rs principles. Res Vet Sci 2019;122:56-63.

4. Martini L, Fini M, Giavaresi G, Giardino R. Sheep model in orthopedic research: a literature review. Comp Med 2001; 51:292-9.

5. Holt JD, Cameron D, Dias N, et al. The sheep as a model of preclinical safety and pharmacokinetic evaluations of candidate microbicides. Antimicrob Agents Chemother 2015;59: 3761-70.

6. Braak H, Braak E, Strothjohann M. Abnormally phosphorylated tau protein related to the formation of neurofibrillary tangles and neuropil threads in the cerebral cortex of sheep and goat. Neurosci Lett 1994;171:1-4.

7. Van Dam D, De Deyn PP. Animal models in the drug discovery pipeline for Alzheimer's disease. Br J Pharmacol 2011;164: 1285-300.

8. Kay GW, Oswald MJ, Palmer DN. The development and characterisation of complex ovine neuron cultures from fresh and frozen foetal neurons. J Neurosci Methods 2006;155:98-108.
9. Roselli CE, Larkin K, Resko JA, et al. The volume of a sexually dimorphic nucleus in the ovine medial preoptic area/anterior hypothalamus varies with sexual partner preference. Endocrinology 2004;145:478-83.

10. Mura A, Gadau S, Lepore G, et al. Expression and distribution of $\mathrm{P} 450$-aromatase in the ovine hypothalamus at different stages of fetal development. Neuro Endocrinol Lett 2010;31:690-9.

11. Lepore G, Gadau S, Mura A, et al. Aromatase immunoreactivity in fetal ovine neuronal cell cultures exposed to oxidative injury. Eur J Histochem 2009;53:233-8.

12. Lepore G, Gadau S, Peruffo A, et al. Aromatase expression in cultured fetal sheep astrocytes after nitrosative/oxidative damage. Cell Tissue Res 2011;344:407-13.

13. Lepore G, Zedda M, Mura E, et al. Brain aging and testosterone-induced neuroprotection: studies on cultured sheep cortical neurons. Neuro Endocrinol Lett 2013;34:395-401.

14. Mura E, Lepore G, Zedda M, et al. Sheep primary astrocytes under starvation conditions express higher amount of LC3 II autophagy marker than neurons. Arch Ital Biol 2014;152:47-56.

15. Farina V, Lepore G, Biagi F, et al. Autophagic processes increase during senescence in cultured sheep neurons and astrocytes. Eur J Histochem 2018;62:22-7.

16. Hill E, Nagel D, Parri R, Coleman M. Stem cell-derived astrocytes: are they physiologically credible? J Physiol 2016;594:6595-606.

17. Grainger AI, King MC, Nagel DA, et al. In vitro Models for Seizure-Liability Testing Using Induced Pluripotent Stem Cells. Front Neurosci 2018;12:590.

18. Logan S, Arzua T, Canfield SG, et al. Studying Human Neurological Disorders Using Induced Pluripotent Stem Cells: From 2D Monolayer to 3D Organoid and Blood Brain Barrier Models. Compar Physiol 2019;9:565-611.

19. Polin RA, Abman SH, Rowitch D, Benitz WE. Fetal and Neonatal Physiology. New York: Elsevier; 2016.

20. McGeady TA, Quinn PJ, Fitzpatrick ES, Ryan MT. Veterinary Embryology. Blackwell, Oxford 2006.

21. Gadau SD. Morphological and quantitative analysis on a-tubulin modifications in glioblastoma cells. Neurosci Lett 2018.

22. Dráberová E, Lukás Z, Ivanyi D, et al. Expression of class III beta-tubulin in normal and neoplastic human tissues. Histochem Cell Biol 1998;109:231-9.

23. Schock SC, Jolin-Dahel KS, Schock PC, et al. Striatal interneurons in dissociated cell culture. Histochem Cell Biol 2010;134:1-12.

24. Hofman MA. Size and shape of the cerebral cortex in mammals. The cortical surface. Brain Behav Evol 1985;27:28-40.

25. Gibbons HM, Dragunow M. Adult human brain cell culture for neuroscience research. The Int $\mathrm{J}$ Biochem Cell Biol 2010;2:844-56.

26. Townes-Anderson E, MacLeish PR, Raviola E. Rod cells dissociated from mature salamander retina: ultrastructure and uptake of horseradish peroxidase. J Cell Biol 1985;100:175-88.

27. Bologa L, Joubert R, Bisconte JC, et al. Development of immunologically identified brain cells in culture: quantitative aspects. Exp Brain Res 1983;53:163-7.

28. Ranganatha N, Kuppast IJ. A review on alternatives to animal testing methods in drug development. Int J Pharm Pharmaceut Sci 2012;4:28-32.

29. Doke SK, Dhawale SC. Alternatives to animal testing: A review. Saudi Pharm J 2015;23:223-9.

30. Price DL, Sisodia SS, Koo EH, et al. Neuronal disorders: studies of animal models and human diseases. Toxicol Pathol 1990;18:128-37. 